\title{
Photo-induced enhanced Raman spectroscopy (PIERS): sensing atomic-defects, explosives and biomolecules
}

D. Glass, E. Cortés, S. Ben-Jaber, T. Brick, R. QuesadaCabrera, et al.

D. Glass, E. Cortés, S. Ben-Jaber, T. Brick, R. Quesada-Cabrera, W. J. Peveler, Y. Zhu, C. S. Blackman, C. R. Howle, I. P. Parkin, S. A. Maier, "Photo-induced enhanced Raman spectroscopy (PIERS): sensing atomicdefects, explosives and biomolecules

," Proc. SPIE 11010, Chemical, Biological, Radiological, Nuclear, and Explosives (CBRNE) Sensing XX, 110100D (17 May 2019); doi: $10.1117 / 12.2518948$

Event: SPIE Defense + Commercial Sensing, 2019, Baltimore, Maryland, United States 


\title{
Photo-induced enhanced Raman spectroscopy (PIERS): sensing atomic-defects, explosives and biomolecules
}

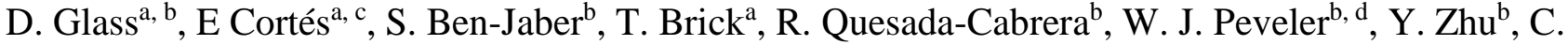 \\ S. Blackman ${ }^{\text {b }, ~ C . ~ R . ~ H o w l e ~}{ }^{\text {e }}$ I. P. Parkin ${ }^{\text {, }, ~ S . ~ A . ~ M a i e r * a, ~ c ~}$ \\ ${ }^{a}$ The Blackett Laboratory, Department of Physics, Imperial College, London, SW7 2AZ, UK; \\ ${ }^{b}$ Department of Chemistry, University College London, 20 Gordon St., London, WC1H 0AJ, UK; \\ ${ }^{c}$ Chair in Hybrid Nanosystems, Nanoinstitute Munich, Ludwig-Maximilians-Universität München, \\ 80799, München, Germany; ${ }^{\mathrm{d} D i v i s i o n}$ of Biomedical Engineering, School of Engineering, \\ University of Glasgow, Glasgow, G12 8LT, UK; ${ }^{~}$ Defence Science and Technology Laboratory, \\ Porton Down, Salisbury, Wiltshire, SP4 0JQ \\ * stefan.maier@physik.uni-muenchen.de; www.hybridplasmonics.org/
}

\begin{abstract}
Enhanced Raman relies heavily on finding ideal hot-spot regions which enable significant enhancement factors. In addition, the termed "chemical enhancement" aspect of SERS is often neglected due to its relatively low enhancement factors, in comparison to those of electromagnetic (EM) nature. Using a metal-semiconductor hybrid system, with the addition of induced surface oxygen vacancy defects, both EM and chemical enhancement pathways can be utilized on cheap reusable surfaces. Two metal-oxide semiconductor thin films, $\mathrm{WO}_{3}$ and $\mathrm{TiO}_{2}$, were used as a platform for investigating size dependent effects of Au nanoparticles (NPs) for SERS (surface enhanced Raman spectroscopy) and PIERS (photo-induced enhanced Raman spectroscopy - UV pre-irradiation for additional chemical enhancement) detection applications. A set concentration of spherical Au NPs (5, 50, 100 and $150 \mathrm{~nm}$ in diameter) was drop-cast on preirradiated metal-oxide substrates. Using 4-mercaptobenzoic acid (MBA) as a Raman reporter molecule, a significant dependence on the size of nanoparticle was found. The greatest surface coverage and ideal distribution of AuNPs was found for the $50 \mathrm{~nm}$ particles during SERS tests, resulting in a high probability of finding an ideal hot-spot region. However, more significantly a strong dependence on nanoparticle size was also found for PIERS measurements completely independent of AuNP distribution and orientation affects - where $50 \mathrm{~nm}$ particles were also found to generate the largest PIERS enhancement. The position of the analyte molecule with respect to the metal-semiconductor interface and position of generated oxygen vacancies within the hot-spot regions was presented as an explanation for this result.
\end{abstract}

Keywords: SERS (surface enhanced Raman spectroscopy), PIERS, oxygen vacancy defects, metal oxide, chemical sensing, enhanced detection

\section{INTRODUCTION}

Raman spectroscopy is commonly used for molecular identification and characterization through the activation of specific molecular vibrational modes (allowing "fingerprint" molecular detection). ${ }^{1-4}$ However, common applications of Raman spectroscopy were only developed in the last 50 years, primarily through the discovery of Surface Enhanced Raman Spectroscopy (SERS). Although originally hindered by the intrinsically low Raman cross-sections of most chemicals, the development of SERS substrates enabled significant spectral enhancements allowing detection of much lower analyte concentrations, resulting in what has now become a powerful analytical technique. ${ }^{5}$ SERS active substrates have recently pushed detection limits towards the single-molecule level ${ }^{5-8}$, thereby opening up the potential for SERS as an instrumental technique for homeland security, chemical analysis and environmental monitoring applications, amongst others.

The precise mechanism behind how SERS operates has long been debated within the literature. Generally, the technique is understood through two enhancement mechanisms; namely electromagnetic (EM) ${ }^{9}$ enhancement and chemical 
enhancement (CE). ${ }^{10}$ The focusing and intensification of EM fields to nanoscale volumes has long known to be possible through noble metal nanostructures via local surface plasmonic effects, originating from collective movement of free electrons within the structures. ${ }^{5}$ When analyte molecules find themselves within such intense fields, referred to as "hotspot" regions, the Raman scattering signal from the molecular vibrations becomes greatly enhanced, normally by a factor of $10^{6}$ or greater. ${ }^{11}$ Charge transfer processes due to vibronic coupling of resonances in the molecule-substrate system provide additional SERS enhancements, ${ }^{12,13}$ often named under the umbrella term of chemical enhancements (CE) and typically enhance by a factor of 10-100. ${ }^{10}$ Substrates which exhibit CEs are often, but not exclusively, semiconductor dielectric substrates. Although CEs are widely accepted as an alternative mechanism, to date EM enhancements through noble metal nanostructures remains the primary SERS mechanism. Yet, by employing a metal-dielectric hybrid system, for example a metal-oxide substrate as a platform for plasmonic metal nanoparticles on the surface, a synergistic SERS enhancement utilizing both mechanisms can be exploited allowing sensitive detection of trace levels of biomolecules, pollutants and explosives.

Recently, Parkin, Maier et al. reported on a novel method for SERS enhancements in these hybrid systems. ${ }^{14}$ An additional chemical enhancement pathway was demonstrated, incorporating photo-induced surface oxygen vacancy states on $\mathrm{TiO}_{2}$ surfaces. It was reported that the oxygen vacancy defects facilitated semiconductor-defect-metal-analyte vibronic couplings, furthering the Raman enhancement. The process was termed "Photo-induced enhanced Raman Spectroscopy", PIERS. Utilizing PIERS as an additional enhancement mechanism for trace level detection, additional studies on both traditional semiconductors ${ }^{15,16}$ and un-conventional SERS substrates ${ }^{17}$ have since been reported. Although within PIERS, resonant vibronic-coupling charge transfers are utilized, hence, a metal-free system would still facilitate semiconductordefect-analyte transfers with a noticeable PIERS enhancement. Yet, due to the low levels of chemical enhancement a primary role of the metal nanoparticle (NP) is to facilitate measuring of the subtle changes in the PIERS enhancement over time through causing a significant time-independent EM enhancement contribution. In addition, metal-semiconductor junctions have been shown to enable effective carrier separation, prolonging carrier lifetimes which in turn may promote vacancy formation. However, further roles of the metal nanoparticle and how the distribution of NPs affect the PIERS measurement are unknown.

Drop casting AuNP solutions, although is a cheap and efficient way of depositing NPs, often results in a large range of possible orientations of the NPs on the surface. In addition, commercial metal-oxide thin films are often found to have significant surface roughness, which can promote nanoparticle aggregation and other negative effects for SERS applications, in contrast to small discrete clusters which are favorable. ${ }^{18}$ Further thermal annealing of the metal-oxides has been shown to smoothen the surface, although a large number of nanoscale cracks and deformations can still be found. Therefore, there are many factors that can affect the resultant position of the AuNPs on the surface. The NP shape, orientation and position on the surface has widely been shown to greatly affect the SERS intensity. ${ }^{13,19-21}$ By using spherical NPs effects due to orientation can effectively be removed. However, a dominant factor in SERS comes from the confined EM hot-spots, due to plasmonic affects in the form of local surface plasmonic resonances (LSPR). The size and strength of the confined field has been shown to largely depend on the distance between NPs on the surface, ${ }^{18,21-23}$ where typical sizes of the hot-spot regions are on the nanoscale. Conversely, if particles are too close together clustering into large aggregations can occur removing some of the field confinement responsible for SERS. It is important to note, within large aggregates effectively cavities can between exterior NPs can form resulting in confined EM fields and hot-spot regions, as with roughened surfaces. However, in comparison to discrete clusters of NPs generally these enhancements are relatively lower, hence ideally are avoided for SERS applications. However, with drop-casting introducing an element of randomness in the resultant position of the NP on the surface, it is possible the particles will not be in ideal SERS arrangements, and therefore result in areas of the substrate where the analyte molecule is masked. Yet with many nanoparticle deposition methods, such as the use of lithographic techniques, often being rather costly, a comprise is needed. The effect of nanoparticle size on the resultant distribution and SERS enhancements for practical dielectric PIERS remains unknown.

In this paper the effect of nanoparticle size on $\mathrm{WO}_{3}$ and $\mathrm{TiO}_{2}$ substrates were investigated for sensing applications using the PIERS technique. Initial SERS studies were investigated to see the effect the size of NP has on SERS intensities. Through imaging and taking a series of Raman scans and maps at positions within and on the edge of a droplet of AuNPs for each size, on each respective metal-oxide, a strong dependence on NP size was found. In addition, by measuring samples after prolonged UV exposure, with induced surface oxygen vacancies, a strong effect of NP size on PIERS enhancements was also found. This rather unexpected result was found to be independent of AuNP distribution, or other 
significant SERS effects, and so was attributed primarily to the position of surface vacancy states within the confined hotspot regions and the influence the effective surface area at the metal-semiconductor interface can have on oxygen vacancy formation.

\section{METHODOLOGY}

\subsection{Substrate synthesis}

$\mathrm{WO}_{3}$ and $\mathrm{TiO}_{2}$ films were synthesized using aerosol-assisted chemical vapor deposition (AA-CVD). For $\mathrm{WO}_{3}$ films, quartz substrates were first cleaned with isopropanol and then subsequently with acetone after which they were dried with air and placed into the CVD reactor. The CVD reactor consisted of a $320 \mathrm{~mm}$-long graphite heating block accommodated within a quartz tube with three Whatman heater cartridges. Pt-Rh thermocouples were used to control the temperature of the entire system. The reactor chamber was initially heated to $300{ }^{\circ} \mathrm{C}$. $\left[\mathrm{W}(\mathrm{CO})_{6}\right](0.06 \mathrm{~g}, 0.17 \mathrm{mmol})$ was dissolved in an acetonemethanol solution (15 ml, 2:1, Sigma Aldrich, $\geq 99.6 \%$ ) and placed in a glass flask in an ultrasonic humidifier (Liquifog, Johnson Matthey operating at $2 \mathrm{MHz}$ ). This was used to form aerosol droplets of a precursor solution which were transported to the heated rector chamber under the flow of nitrogen gas $\left(B O C, 300 \mathrm{~cm}^{3} \mathrm{~min}^{-1}\right)$. The tungsten solution was allowed to pass over the quartz substrates in the reactor for $30 \mathrm{mins}$, thereafter the reactor was allowed to cool to room temperature. The substrates were then annealed at $500{ }^{\circ} \mathrm{C}$ for 2 hours in air, at a heating rate of $5{ }^{\circ} \mathrm{C} \mathrm{min}^{-1}$, and subsequently allowed to cool back to room temperature.

A similar cleaning and deposition method was employed for $\mathrm{TiO}_{2}$ substrates. However, the significant changes in the procedure are as follows. Titanium (IV) isopropoxide (Sigma Aldrich, $97 \%, 0.5 \mathrm{ml}$ ) in methanol (Fisher Scientific HPLC grade, $20 \mathrm{ml}$ ) was used as precursor and solvent respectively. The gas flow rate was set to $1 \mathrm{~L} \mathrm{~min}^{-1}$. $\mathrm{TiO}_{2}$ films were initially deposited at $500{ }^{\circ} \mathrm{C}$ and subsequently annealed to $1000{ }^{\circ} \mathrm{C}$ in air for 10 hours and then allowed to cool to room temperature to obtain a pure Rutile phase. This was confirmed by X-ray diffraction and Raman spectroscopy, where no traces of anatase were detected.

\subsection{Chemical and substrate preparation}

Each metal-oxide substrate was placed under a UV lamp (UVItec LI 215G model $(\lambda=254 \mathrm{~nm}$ )) at a rough distance of 1 $\mathrm{cm}$ between the lamp and substrate under ambient conditions, giving a resultant intensity between 12-15 $\mathrm{mW}$. After 30 minutes of UV exposure to the substrates the UV lamp was switched off and aqueous spherical AuNP solutions of either 5 (Sigma), 50 (Aldrich), 100 (Aldrich) or 150 (BBI Solutions) $\mathrm{nm}$ in diameter were deposited on the substrate $(0.15 \mathrm{~mL})$. The lamp was then switched back on until the droplet had completely dried, typically 5 minutes. For SERS measurements the samples were then removed from the UV lamp and kept in the dark under ambient conditions for an hour. For PIERS measurements the samples were left for an additional 15 minutes of UV irradiation (a combined total of 45 minutes).

4-mercaptabenzoic acid (MBA, Sigma Aldrich) was dissolved in ethanol (VWR Chemicals) to create a $1 \times 10^{-5} \mathrm{M}$ solution. $0.15 \mathrm{ml}$ of the MBA solution was drop cast onto the metal-oxide substrates (with and without AuNPs) and excess solvent left to evaporate, typically 10-30 seconds. The substrates were then immediately placed under the Raman microscope for analysis.

For SEM images, a thin layer of pure Au $(5 \mathrm{~nm})$ was sputtered on top of the metal-oxide substrates (with and without AuNPs) using a Q150T S sputter system under an Ar environment.

\subsection{Characterization of substrates}

Raman studies were conducted using a confocal Raman microscope (WiTec) equipped with a He-Ne laser $(\lambda=633 \mathrm{~nm})$ with a bright field objective (Zeiss 100x NA 0.9) at an average power of $3 \mathrm{~mW}$. The acquisition time for individual spectra and line-scans was 1 seconds where 4 consecutive measurements were averaged for each spectrum. For Raman maps acquisition time was reduced to 0.5 seconds where 75 positions were taken for 75 lines over a $10 \mathrm{x} 10 \mu \mathrm{m}$ square. For PIERS measurements Raman spectra were recorded at multiple positions across on the surface, as described previously, after which the position of the laser was moved significantly far away from the measured positions. The system was left in place at the same focus and laser power for an hour, to allow the return to the conventional baseline, whereupon a second Raman spectrum was taken at each measured position to determine the PIERS enhancement contribution solely due to induced vacancy states. 
$\mathrm{X}$-ray diffraction (XRD) analysis was carried out using a Bruker-Axs D8 (Lynxeye XE) diffractometer. A mono-chromated copper $\mathrm{X}$-ray source $\left(\mathrm{K} \alpha_{1}, \lambda=1.54 \AA\right)$ was used under a glancing incident angle $(\theta)$ of $1^{\circ}$.

Scanning electron micrographs (SEM) were taken at $10 \mathrm{kV}$ acceleration voltage, a working distance of $7 \mathrm{~mm}$ and an aperture of $30 \mu \mathrm{m}$ using a Raith e-line system.

\section{RESULTS AND DISCUSSION}

Thin films of $\mathrm{WO}_{3}$ (mono-clinic) and $\mathrm{TiO}_{2}$ (rutile) were grown via aerosol-assisted chemical vapor deposition (AA-CV) on quartz substrates, as described above. Substrates were exposed to UV irradiation for 30 minutes where subsequently solutions of Au nanoparticles (NP) $(5,50,100$ and $150 \mathrm{~nm})$ were drop cast upon the thin films. The deposited solution was left to dry under UV irradiation. The crystal structure of the prepared films studied was confirmed using $\mathrm{X}$-ray diffraction (XRD), Figure 1. Peak characteristics of diffraction patterns for both metal-oxide films were confirmed with reported spectra in the literature and XRD databases.

a)

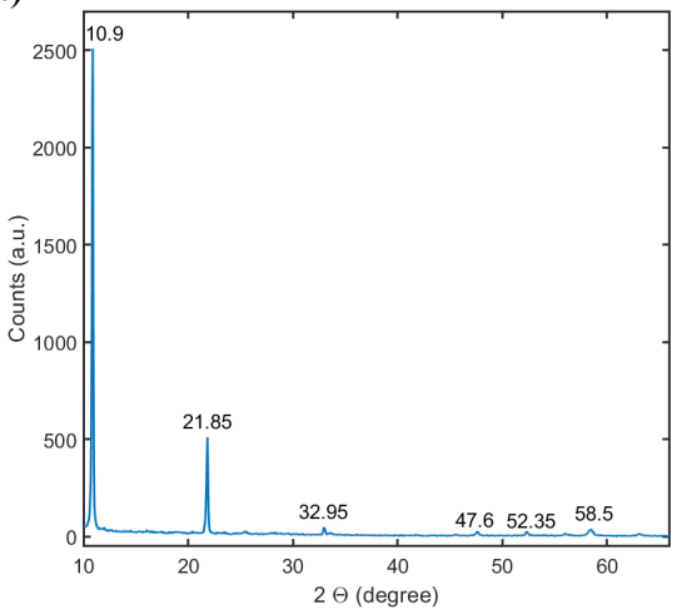

b)

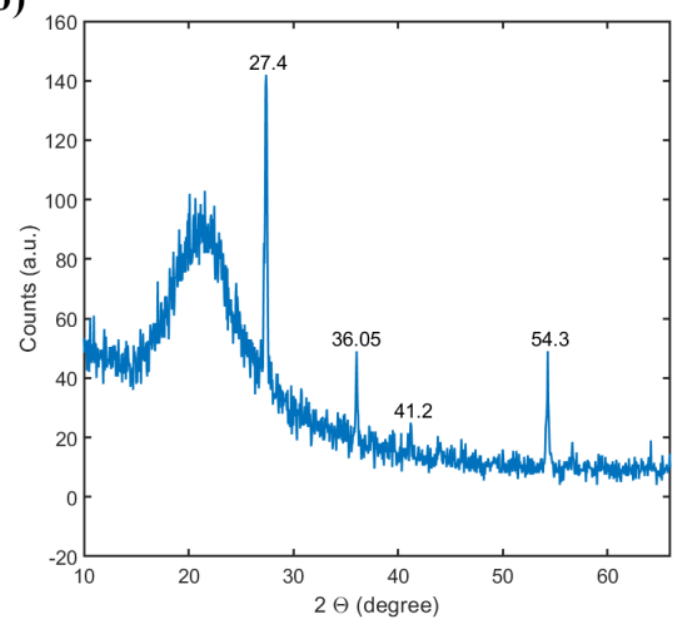

Figure 1. XRD patterns for a) $\mathrm{WO}_{3}$ and b) $\mathrm{TiO}_{2}$ thin films.

4-mercaptobenzoic acid (MBA) was drop cast onto the films prior to Raman analysis. For SERS measurements the films were left in the dark for an hour, or more, to ensure surface oxygen vacancy induced during the nanoparticle deposition ${ }^{14}$ and solution drying were completely healed prior to Raman measurements. Both metal-oxide substrates were found to have characteristic Raman bands for each respective material, observed at 78, 263, 768 and $939 \mathrm{~cm}^{-1}$ for $\mathrm{WO}_{3}$ and 438 and $602 \mathrm{~cm}^{-1}$ for $\mathrm{TiO}_{2}$ respectively, see Figure 2. $\mathrm{WO}_{3}$ and $\mathrm{TiO}_{2}$ films with $\mathrm{MBA}$ deposited with no AuNPs were not found to show any Raman bands corresponding to MBA at any measured position (Figure 2a) for both SERS and PIERS spectra. This was assumed to be due to low Raman cross sections in combination with low concentration of MBA used in the study. Figure 2a also shows an average Raman spectrum for MBA powder taken as a reference. Although 8 noticeable bands are present in the bulk spectra, due to restrictions of molecular orientation and the vibrational limitations on a surface only 2 bands were found for SERS measurements, namely bands at $1065 \mathrm{~cm}^{-1}$ and $1575 \mathrm{~cm}^{-1}$ respectively. These were attributed to $v 8$ and $v 12$ aromatic ring vibrations respectively. ${ }^{24}$ As no bands for the metal-oxides were found around these regions, the appearance of both these bands, 1065 and $1575 \mathrm{~cm}^{-1}$, simultaneously in spectra was used to identify the presence of MBA.

As discussed above, although drop-casting NPs is a cost-effective way of depositing NPs, it results in random orientations and a random distribution of the NPs, with large numbers of particles found at the edge of the dried ring. In addition to distribution affects resulting from the surface roughness, even an average of 10-20 single random spectra are not representative of the average SERS enhancement for each respective NP size for each respective metal-oxide. Hence, for each particle size on each respective substrate an average of over 100 spectra were taken. It should be noted that only spectra at positions on the surface where MBA was found were included in determining an average spectrum. 
a)

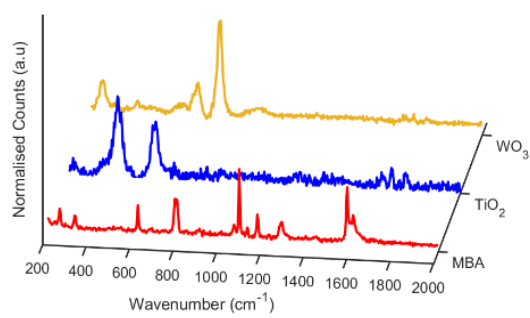

b)

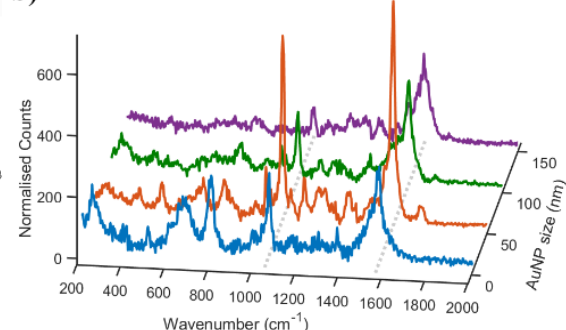

c)

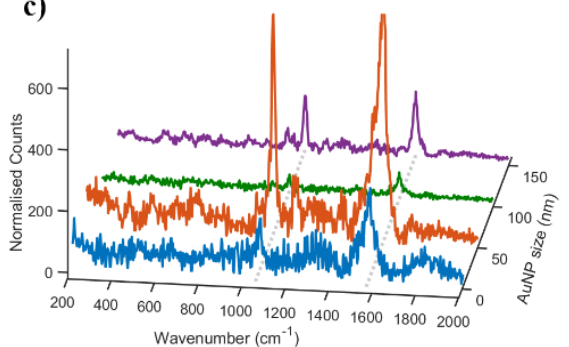

Figure 2. a) Raman spectra for $\mathrm{WO}_{3}$ and $\mathrm{TiO}_{2}$ with $\mathrm{MBA}$ deposited on and MBA powder. Notably with no AuNPs present no MBA bands are visible and only bands corresponding to the respective substrates can be seen. Average Raman spectra for MBA on b) $\mathrm{WO}_{3}$ and c) $\mathrm{TiO}_{2}$ thin films for 5, 50, 100 and $150 \mathrm{~nm}$ sized Au NPs. Raman bands for MBA were found on all substrates with AuNPs with significantly large SERS enhancements found using $50 \mathrm{~nm}$ particles, relative to the other sizes.

Figure 2 shows noticeable enhancements of the MBA bands were found for every size of NPs chosen. However, it is clearly shown that the size of NP was found to considerably change SERS enhancement for $\mathrm{MBA}$ on both $\mathrm{WO}_{3}$ and $\mathrm{TiO}_{2}$. Similar average enhancements for 5, 100 and $150 \mathrm{~nm}$ sized particles were found on $\mathrm{WO}_{3}$ substrates. For TiO films, the average enhancement for 5 and $150 \mathrm{~nm}$ sized particles was found to be similar in magnitude, whereas the $100 \mathrm{~nm}$ Au particles were found to give a considerably lower enhancement. However, for both $\mathrm{WO}_{3}$ and $\mathrm{TiO}_{2}$ films the largest enhancement in Figure 2 was found with $50 \mathrm{~nm}$ AuNPs. Hence, the enhancement of the analyte molecule significantly depends on the size of the NP used for SERS.

As described above, the primary mechanism for SERS enhancement with AuNPs relies on plasmonic electromagnetic field enhancements. With drop-casting resulting in a large and random distribution of inter-nanoparticle distances the average SERS enhancement shown is likely to primarily depend on this factor. Yet, other significant processes may also help explain the size dependency of the SERS enhancement. It is well known that by changing the size of the NP the local surface plasmon resonance can shift in frequency. ${ }^{25,26} \mathrm{~A}$ shift in the peak absorption frequency can affect the resonant transfer between the NP and the Raman probe laser, resulting in a large enhancement change. In addition, the interaction between the surfaces of the metal NP and semiconductor substrate may change with nanoparticle size, as less or more surface area is in contact between the metal and semiconductor. Furthermore, the position of the analyte probe molecule on the surface of the metal-oxide and nanoparticle is known to affect the SERS enhancement. Plasmonic assisted SERS heavily relies on hot-spot regions of large EM enhancements. Hence, if a molecule lies within this hot-spot region a large SERS signal can be seen. Conversely, if the analyte molecule orientates itself or binds outside the hot-spot regions, a much lower or no enhancement will be seen. The results shown in Figure 2 represent a combination of all these factors. 5 nm particles are likely to be too small to have an effective molecule-nanoparticle interaction for SERS applications. Although the large sizes of 100 and $150 \mathrm{~nm}$ particles allow more molecule-nanoparticle interactions, the significant decrease in surface area could explain the lower enhancements seen. The largest average enhancement in Figure 2, suggesting that a possible optimum nanoparticle size placed on top of the metal-oxides surfaces, is for $50 \mathrm{~nm}$ AuNPs.

For commercial chemical sensing substrates, in addition to the SERS enhancement factor the likelihood of finding a hotspot region plays an important role. A series of Raman spectra were recorded across the surfaces of each respective metaloxide, for each size of NPs. The spectra were taken over the edge of the AuNP droplet in order to differentiate enhancements in band intensities of MBA arising from the AuNPs and from the metal-oxide substrates respectively. Optical and SEM images of the AuNP droplet edge can be seen in Figure $3 \mathrm{~b}$ and $3 \mathrm{c}$ respectively. It is important to note, as described above, that the results shown here primarily reflect the relative distribution of each size of NP, as oppose to the possible enhancement for each respective size. Although one size may theoretically be able to confine stronger fields and therefore may be assumed falsely to be the ideal size, for practical SERS and PIERS applications to reliably form hotspot regions on the substrate is equally or more desirable. Figure 3a shows clearly no band intensities corresponding to MBA were found outside the AuNP droplets, as expected due to the very low SERS enhancements from dielectrics and results shown in Figure 2a. Figure $3 d$ shows an enlarged part of Figure 3a, results found within the black lined square. It is clear that Figure 3a (and Figure 3d) show how MBA was found for each size of NP, although not at every position measured on $\mathrm{TiO}_{2}$. Similar results were found for $\mathrm{WO}_{3}$ films, this is not shown. Notably, low enhancements were noticed for $100 \mathrm{~nm}$ particles during the measurements, with very few sampled positions showing hot-spot regions. Although both $5 \mathrm{~nm}$ and $150 \mathrm{~nm}$ particles show a few positions of significant enhancement, again very few hot-spot regions were found 
across the surface. On the other hand, Figure 3a shows considerable enhancements at nearly every measured position for $50 \mathrm{~nm}$ particles, noticeably higher than all the other sizes. Many measured positions using $50 \mathrm{~nm}$ were found to be over 10x the enhancement, relative to the other particle sizes. A size dependence for SERS enhancements was also found by Bell et al. ${ }^{27}$, where thiophenol was used as a test compound. The results were primarily explained to be due to shifts in plasmon absorption with particle size, agreeing well with the results presented here.
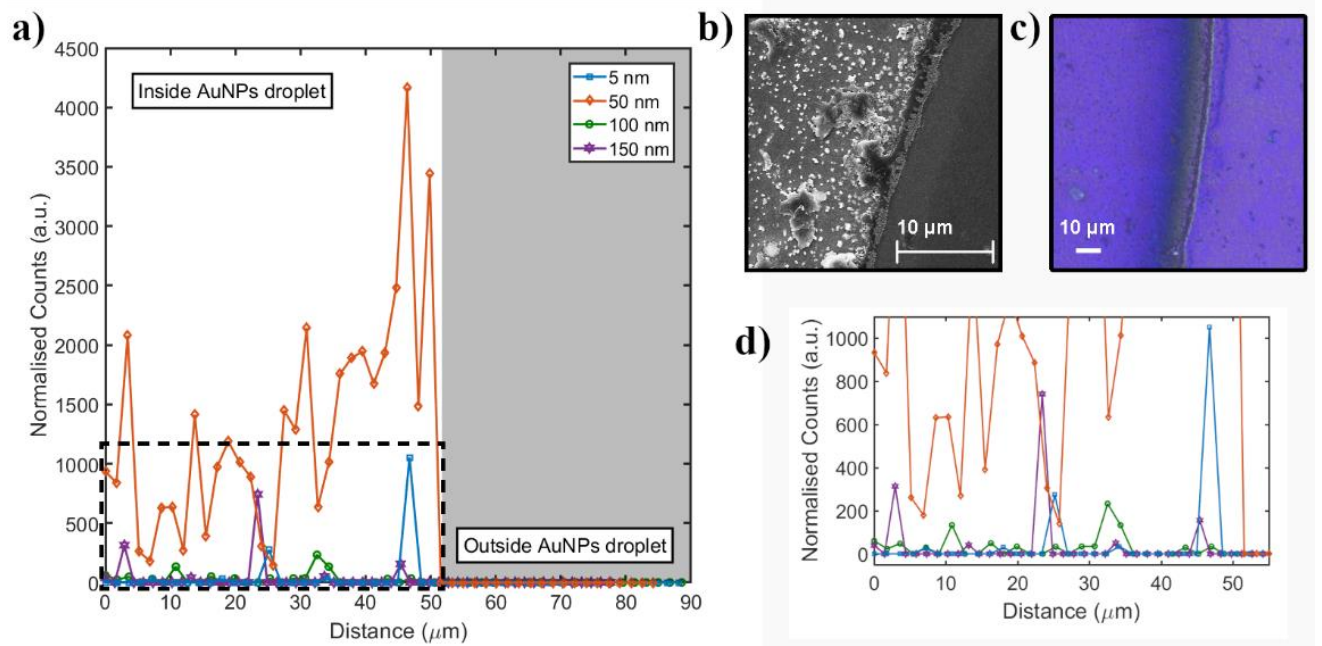

Figure 3. a) Normalized series Raman band intensity for a peak assigned to MBA $\left(1570 \mathrm{~cm}^{-1}\right)$ across the surface of TiO 2 with different sized NP on at the edge of the deposited AuNP droplet. Sample SEM image (b) and optical image (c) of AuNP droplet edge on metal-oxide surface. d) Enlarged normalized Raman band intensities shown in part a) (black lined box). Note, although MBA was deposited across the whole surface no Raman bands for MBA were found outside the AuNP droplet for every sample.
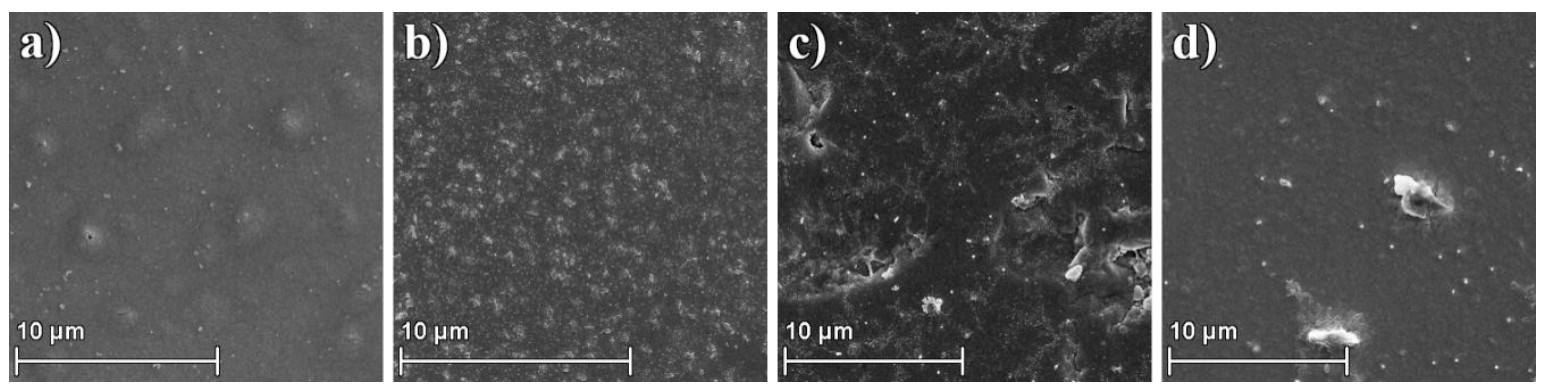

Figure 4. Sample SEM images of $\mathrm{WO}_{3}$ surfaces with a) $5 \mathrm{~nm}$, b) $50 \mathrm{~nm}$, c) $100 \mathrm{~nm}$ and d) $150 \mathrm{~nm}$ AuNPs deposited on. The white "dots" in each image correspond to the position of the nanoparticles on the surface. Each AuNP size shows relatively poor NP distribution, excluding b) (50 nm NPs) which are visibly presented in greater numbers per area measured. Note, for a) the visible dots were found to represent clusters of $5 \mathrm{~nm}$ particles, as opposed to individual particles.

SEM images of each substrate were taken to compare the size distributions of the nanoparticles to the Raman results found in addition to determining the size of the nanoparticles, Figure 4. The average size of the nanoparticle found with each AuNP solution was found to be around the values presented in this work (5, 50 100 and $150 \mathrm{~nm}$ respectively). The shape of each AuNP solution was confirmed to be predominantly spherical upon larger magnifications. However, interestingly within the distributions of $150 \mathrm{~nm}$ AuNP on each metal-oxide surface the occasional triangular particle was found to be visible within a cluster of spherical particles, images not shown. These were found to represent such a small fraction of the deposited particles that Raman enhancement effects from the triangular particles were presumed to be insignificant. A significant number of positions imaged over both metal-oxide surfaces were found to contain a large number of clustered NPs. These clusters generally do not exhibit as strong LSPR effects and therefore do not positively help towards SERS applications, as described above. Figure 4 shows a representative sample image from positions across $\mathrm{WO}_{3}$ substrates with each respective AuNP size. Figure 4a, 4c and $4 \mathrm{~d}$ all show considerably large distance between the AuNPs (or small clusters of a few particles). This supports the Raman results where large distances (e.g. $10 \mu \mathrm{m}$ ) were found between noticeable 
Raman intensities, Figure 3. The diffraction limit of the Raman laser used was calculated to be around $0.35 \mu \mathrm{m}$. Assuming the size of the Raman laser was slightly larger than the diffraction limit (as seen in optical images), we can assume around a $1 \mu \mathrm{m}$ spot (or smaller) is sampled at any one time. From SEM images it can be shown that an average area of this size contains a small number of NPs at ideal distances for EM hot-spots on substrates with 5, 100 and $150 \mathrm{~nm}$ particles. Noticeably, Figure 4b shows many more AuNPs were found within the same sampled area, often as single particles or in pairs or with 3 particles together. Distributions of this type are known to result in more focused EM field and larger EM field enhancements, ${ }^{22}$ which results in a more enhanced SERS signal, as found in Figure 3 for $50 \mathrm{~nm}$ particles.

A Raman spectral series of the the metal-oxides surfaces shown in Figure 3 were taken over a relatively large distance (around $85 \mu \mathrm{m}$ ). However, although this may shed light on how practical the use of each sized NP may be on a large scale, at the edge of the AuNP droplet, generally, a larger number of AuNPs can be found. In order to gain a better understanding of the nanoparticle size dependency of SERS enhancements a series of Raman maps were taken at a central region within the AuNP drop. Figure 5 shows sample Raman maps of each NP size investigated on $\mathrm{WO}_{3}$ substrates, where height in $\mathrm{z}$ direction is representative of the normalized Raman band enhancement for MBA (the $1065 \mathrm{~cm}^{-1}$ band). In comparison to the series of line spectra (Figure 3a) many more Raman bands for MBA were found on the Raman map for 5 and $100 \mathrm{~nm}$ particles. However, as the line-scan is taken over larger distances it is possible for the measured spectra at each position to be an average of the local area. On all maps in Figure 5 it is noticeable that with only a small change in $\mathrm{x}$ or $\mathrm{y}$ one may not notice a hot-spot in close proximity. This can explain the apparent discrepancies between the Raman maps and line-scans. The average intensity of the Raman bands by mapping were slightly higher in magnitude than the line-scan average intensity for 5 and $100 \mathrm{~nm} \mathrm{NP}$, however the line-scan averages result from an average of many more spectra and for a longer period of time, which can result in a greater decrease in the measured intensity due to photobleaching effects. Yet, it is noticeable that there are some regions on the sample, as shown by Figure 5a and 5c where significant SERS intensity can be still be found even for the lower acquisition times. Figure $5 \mathrm{~d}$ shows for $150 \mathrm{~nm}$ particles only 1 significant position showed a SERS enhancement. As shown in Figure 4d, this is likely to result from such wide distances between AuNPs and the much smaller area sampled for the Raman map. In contrast to the other AuNP sizes, Figure 5b shows more intense Raman bands were found for $50 \mathrm{~nm}$ AuNPs. Unlike above in Figure 3a, the Raman map for $50 \mathrm{~nm}$ shows many positions on the surface present no MBA Raman bands, however this follows the same explanation above for both 5 and $100 \mathrm{~nm}$ particles. The SEM image shown in Figure $4 \mathrm{~b}$ does show that within the center of the droplet there are regions of the surface where no AuNPs are found. Although it was found to be highly likely to find a hot-spot region when randomly choosing positions within the center of the AuNP droplet for $50 \mathrm{~nm}$ particles. Hence, we can explain the apparent discrepancy between the Raman map and line-scan for $50 \mathrm{~nm}$ particles as follows; the line-scan taken in Figure 3 was conducted over the edge of the NP droplet, higher concentrations of NPs are present and so more positions sampled were found to have enhanced MBA bands.

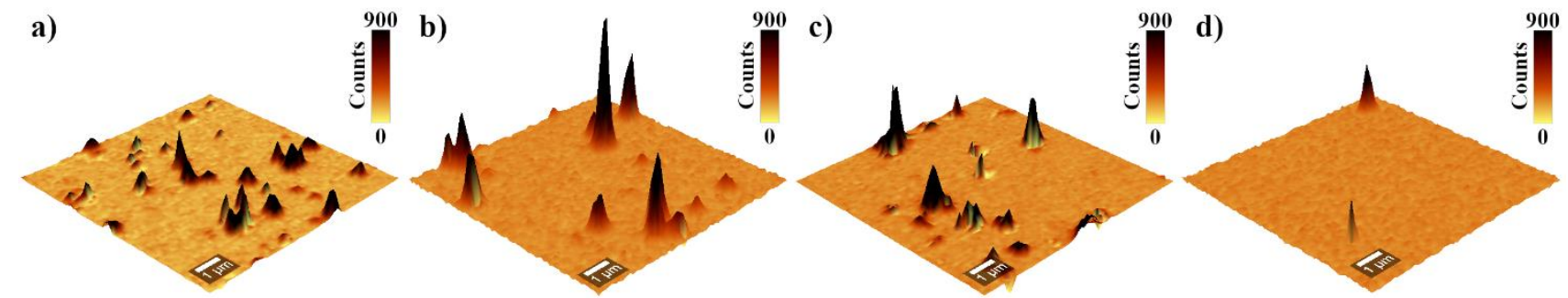

Figure 5. Sample 3D Raman map of MBA (1065 $\mathrm{cm}^{-1}$ band) deposited on a $\mathrm{WO}_{3}$ surface with a) $5 \mathrm{~nm}$, b) $50 \mathrm{~nm}$, c) $100 \mathrm{~nm}$ and d) $150 \mathrm{~nm}$ AuNPs. Heights of the peaks shown correspond to band intensities. Note color maps shown correspond to relative band intensity within each map, whereas peak heights are normalized across the maps.

For PIERS studies, the metal-oxide substrates were under UV exposure for an additional 15 minutes (total of 45 minutes continuously) to ensure maximum vacancy production. After removing from UV exposure, MBA was deposited and Raman spectra recorded immediately. Both Raman bands corresponding to MBA were found to be enhanced above the standard SERS band intensity on both $\mathrm{WO}_{3}$ and $\mathrm{TiO}_{2}$ films. To determine enhancement effects only corresponding to the induced vacancies and not enhancements due to AuNPs (the SERS baseline), PIERS enhancements were determined relative to the SERS intensity at the same position. Therefore, a PIERS enhancement of 1 in Figure 6a corresponds to just a SERS enhancement without any vacancies present. As reported previously, noticeable enhancement differences were 
found between $\mathrm{WO}_{3}$ and $\mathrm{TiO}_{2}$ substrates, with much higher relative PIERS enhancements found on $\mathrm{WO}_{3}$ substrates on average, Figure 6a. For $\mathrm{TiO}_{2}, 5$ and $150 \mathrm{~nm}$ particles found little to no PIERS enhancement, with the average Raman intensity found to be of a similar value to the respective SERS intensity. $100 \mathrm{~nm}$ particles did show a noticeable PIERS enhancement on $\mathrm{TiO}_{2}$ substrates; however, this was found to be much smaller than the PIERS enhancement using $50 \mathrm{~nm}$ particles where the PIERS intensity for $50 \mathrm{~nm} \mathrm{NPs}$ on $\mathrm{TiO}_{2}$ was found to be almost double the SERS. On $\mathrm{WO}_{3}$ substrates, $150 \mathrm{~nm}$ particles also showed a negligible PIERS enhancement. Yet, for all other sizes of NP, much greater PIERS enhancements were found in comparison to the same size $\mathrm{NP}$ on $\mathrm{TiO}_{2}$ substrates. 5 and $100 \mathrm{~nm}$ particles showed a 2- and 5- fold increase in the SERS band intensity, respectively. As with $\mathrm{TiO}_{2}, 50 \mathrm{~nm}$ particles showed the greatest enhancement, almost 9 times the average SERS intensity.
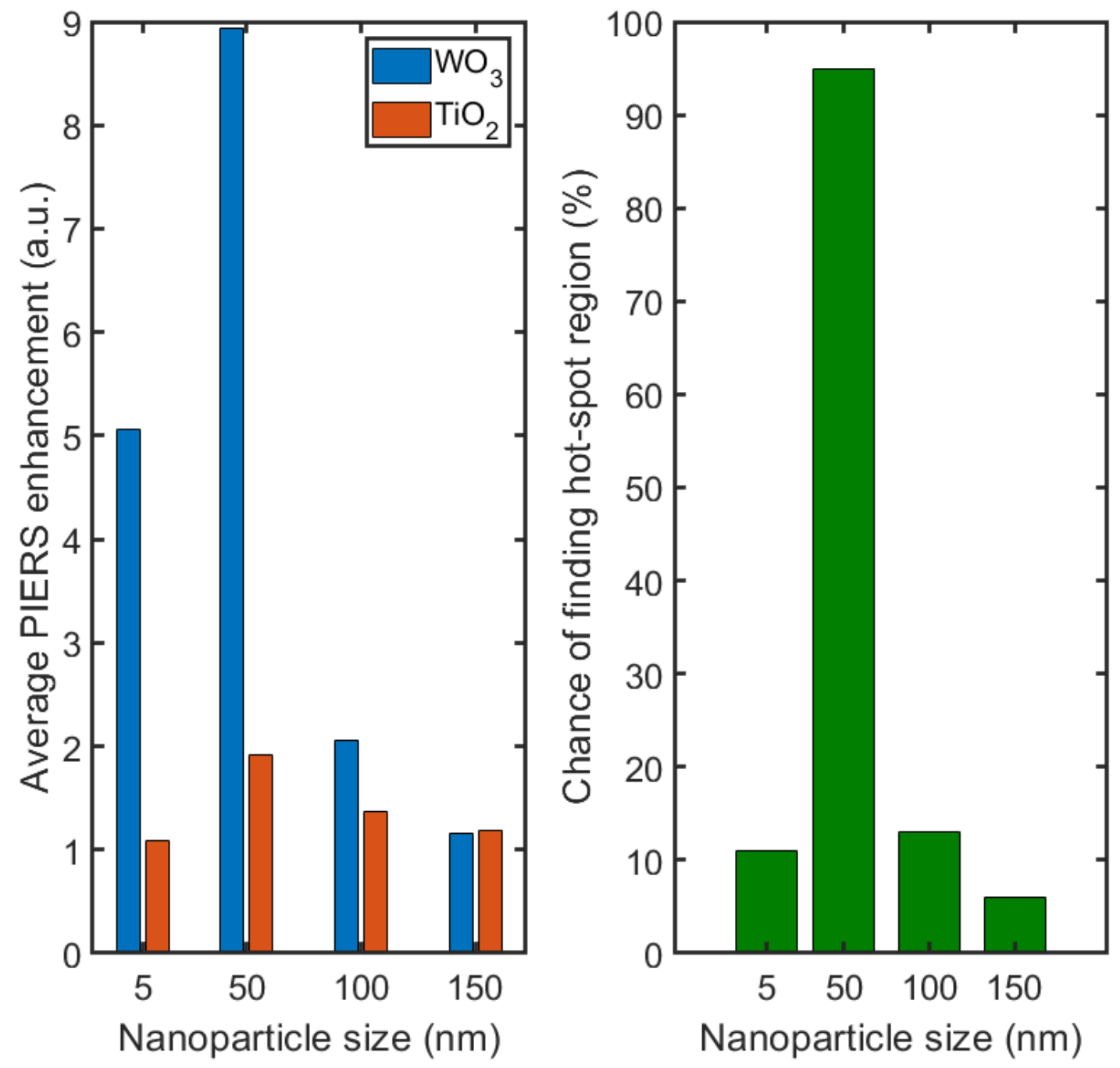

Figure 6. a) Average PIERS enhancement found for each size of $\mathrm{AuNP}$ on $\mathrm{WO}_{3}$ and $\mathrm{TiO}_{2}$, relative to the average SERS enhancement. b) Estimate probability of finding a hot-spot region for each size NP determined from number of MBA bands found during Raman spectra, line-scans and Raman maps for SERS substrates and random Raman scans during PIERS measurements

By sampling many random positions over the metal-oxide surfaces with AuNPs on, over 150 positions, around the center and edge of AuNP droplets, an estimated probability of experimentally finding analyte molecules was determined, Figure $6 \mathrm{~b}$. The recorded percentage chance of finding MBA was based upon all the SERS and PIERS measurements taken (Raman maps and line-scans in addition to random individual spectra recorded). Although an observable Raman enhancement for MBA on both $\mathrm{WO}_{3}$ and $\mathrm{TiO}_{2}$ was found for every size of $\mathrm{NP}$, in practical sensing applications a large enhancement value 
is meaningless for a non-reproducible substrate or with a low probability of finding a position of notable enhancement. 5, 100 and $150 \mathrm{~nm}$ NPs on both $\mathrm{WO}_{3}$ and $\mathrm{TiO}_{2}$ substrates both showed similar trends with regards to the low chance of finding an MBA SERS or PIERS enhancement. Contrastingly, on average a considerable Raman band intensity was always found when sampled randomly using $50 \mathrm{~nm}$ particles. Although Figure $5 \mathrm{~b}$ shows there are positions on the surface within the AuNP droplet where no SERS and as a result no PIERS intensity was detected, this was found to be the case infrequently. Figure 4b, and other SEM images not shown, show there were areas of the sample with few or no AuNPs present, within the AuNP droplet. However, when taking into account the size of the focused Raman laser, on average every position measured statistically contains multiple hot-spots, and so even within the "bare" regions the chances of not sampling any AuNPs was very low. The considerable change in probability between NP size may also explained due to the other mechanism described above. Namely, shifts in LSPR resonances over the different sized NPs used within this study and the importance of the position and placement of the MBA molecule on either the metal-oxide surface or AuNP, which are all known to have an affect on the SERS intensity. Hence, the results may suggest an ideal compromise between available interfacial surface area and likeliness of the analyte molecule reaching the interfacial region can be found with $50 \mathrm{~nm}$ AuNPs.

Overall, a clear NP size dependence on SERS and PIERS enhancements has been shown. Yet despite this, Parkin, Maier et al. ${ }^{14}$ report the basis of the PIERS phenomena arising from surface oxygen vacancy states, which should be independent of nanoparticle size. Notably, the PIERS results reported in Figure 6a are relative to SERS baseline, hence are normalized with respect to positions of the analyte molecule and AuNP distribution on the substrate. As a result, the changes in PIERS intensities must result from changes in oxygen vacancy concentrations around the measured positions as opposed to other SERS effects. The general understanding in the literature of how UV induces surface oxygen vacancies relies on adsorbed molecules forming radicals on the metal-oxide semiconductor surfaces, after light-induced photocatalytic interactions. The formed radicals subsequently interact with the metal-oxide surface, resulting in vacancy formation. As described above, under ambient conditions oxygen and water vapor present in the air may interact with the surface vacancies, effectively healing the defect state. This mechanism appears to be independent of the AuNP, and so must be explained based on the results found in this study. The plasmonic nature of the NPs and metal-semiconductor Schottky barrier allows more efficient carrier separation and in turn has been found to enhancement to photocatalytic potential of the metal-oxide semiconductors. This has been reported to increase the carrier lifetimes, ${ }^{28}$ which in turn can promotes an increased number of radical species and in turn oxygen vacancies. The interfacial surface area at the metal-semiconductor interface could affect the number of charge carriers that can be effectively separated due to interfacial metal-semiconductor plasmonic coupling, ${ }^{29}$ and as a result the resultant number of vacancies available for PIERS enhancements. Furthermore, the position of the vacancy state may play a significant role in the PIERS mechanism, perhaps allowing effective resonant vibronic coupling only when the analyte molecule is placed close enough to both the metal-semiconductor interface and oxygen vacancy. This may largely depend on the ability for other adsorbed molecules on the surface to reach the metalsemiconductor interface or hot-spot region. Hence, the need for each species to be within the EM hot-spot region at the right position, can be significantly be affected by the AuNP size, granting an explanation for NP size dependency of the PIERS technique. To investigate and understand this further, simulations are likely to be required.

\section{CONCLUSION}

Two metal-oxide semiconductor thin films, $\mathrm{WO}_{3}$ and $\mathrm{TiO}_{2}$, were used as a platform for investigating size dependent effects of AuNPs for SERS and PIERS applications for 5, 50100 and $150 \mathrm{~nm}$ sized particles. Through a series of Raman scans at the edge and center of a droplet of AuNPs on each substrate, a strong dependence on NP size was found. Pre-irradiation of the metal-oxide substrates prior to NP deposition was used in an attempted to encourage effective distribution of the NPs whilst keeping the deposition method relatively cheap, for practical applications. Comparing optical and SEM images with Raman mapping and Raman line-scans, the significant difference in SERS enhancements found for each size of NP was explained by the resultant distribution of each respectively sized NPs on the semiconductor surface. Many of the 50 $\mathrm{nm}$ particles were found to be randomly deposited consistently in preferred distributions, enabling many hot-regions to be found and a high probability of finding the analyte molecule. Although SERS enhancements of the Raman reporter were found on both substrates for every size of NP, an additional factor due to resonant Raman - between the Raman laser and LSPR for $50 \mathrm{~nm}$ particles - may have an additional effect causing an increased signal enhancement for $50 \mathrm{~nm}$ particles.

Additionally, an equally strong dependence on NP size was found for PIERS, substrates with induced surface oxygen vacancies present due to additional UV pre-irradiation. Relative enhancements were determined, considering all SERS contributions. Hence, the oxygen vacancy must play a primary role to explain the differences between each PIERS 
substrate, for each NP particle size respectively. As the generally understood mechanism in the literature for vacancy formation and healing is independent of the AuNPs, an explanation based on the size of the NP and ability for the analyte molecule (and surface vacancy) to reside close to the metal-semiconductor interface was presented. Further work on the interaction of molecules around the metal-semiconductor interface may shed light into this explanation and further explain the PIERS dependency on NP size.

\section{REFERENCES}

1 B. Tolaieb, C. J. L. Constantino and R. F. Aroca, Analyst, 2004, 129, 337.

2 E. H. Witlicki, S. Bähring, C. Johnsen, M. V. Solano, K. A. Nielsen, D. W. Silverstein, C. W. Marlatt, L. Jensen, J. O. Jeppesen and A. H. Flood, Chem. Commun., 2017, 53, 10918-10921.

3 F. Yan and T. Vo-Dinh, Sensors Actuators, B Chem., 2007, 121, 61-66.

4 S. S. Ben Jaber, W. J. Peveler, R. Quesada Cabrera, C. W. O. Sol, I. Papakonstantinou and I. P. Parkin, Nanoscale, 2017, 9, 16459-16466.

5 E. C. Le Ru, E. Blackie, M. Meyer and P. G. Etchegoin, J. Phys. Chem. C, 2007, 111, 13794.

6 J. P. Camden, J. A. Dieringer, Y. Wang, D. J. Masiello, L. D. Marks, G. C. Schatz and R. P. Van Duyne, J. Am. Chem. Soc., 2008, 130, 12616.

$7 \quad$ S. Nie and S. R. Emory, Science (80-. )., 1997, 275, 1102.

$8 \quad$ S. Schlücker, Angew. Chemie Int. Ed., 2014, 53, 4756-4795.

9 M. Moskovits, Rev. Mod. Phys., 1985, 57, 783-826.

10 D. P. Fromm, A. Sundaramurthy, A. Kinkhabwala, P. J. Schuck, G. S. Kino and W. E. Moerner, J. Chem. Phys., 2006, 124, 061101.

11 B. Sharma, R. R. Frontiera, A.-I. Henry, E. Ringe and R. P. Van Duyne, Mater. Today, 2012, 15, 16-25.

12 J. R. Lombardi and R. L. Birke, J. Phys. Chem. C, 2014, 118, 11120-11130.

13 I. Alessandri and J. R. Lombardi, Chem. Rev., 2016, 116, 14921-14981.

14 S. Ben-Jaber, W. J. Peveler, R. Quesada-Cabrera, E. Cortés, C. Sotelo-Vazquez, N. Abdul-Karim, S. A. Maier and I. P. Parkin, Nat. Commun., 2016, 7, 12189.

15 Y. Liu, J. Zhu, Y. Wu, M. Zhang, T. Chen and J. Liu, ChemNanoMat, 2019, 5, 55.

16 R. M. Al-Shammari, M. A. Baghban, N. Al-Attar, A. Gowen, K. Gallo, J. H. Rice and B. J. Rodriguez, ACS Appl. Mater. Interfaces, 2018, 10, 30871.

17 S. Almohammed, F. Zhang, B. J. Rodriguez and J. H. Rice, Sci. Rep., 2018, 8, 41-44.

18 J. M. Romo-Herrera, R. A. Alvarez-Puebla and L. M. Liz-Marzán, Nanoscale, 2011, 3, 1304-1315.

19 F. Tian, F. Bonnier, A. Casey, A. E. Shanahan and H. J. Byrne, Anal. Methods, 2014, 6, 9116-9123.

20 W. J. Peveler, S. Ben Jaber and I. P. Parkin, Forensic Sci. Med. Pathol., 2017, 13, 490-494.

21 S. A. Maier, Plasmonics : fundamentals and applications, Springer, 2007.

22 S. L. Kleinman, R. R. Frontiera, A.-I. Henry, J. A. Dieringer and R. P. Van Duyne, Phys. Chem. Chem. Phys., $2013,15,21-36$.

23 C. M. Aikens, L. R. Madison and G. C. Schatz, Nat. Photonics, 2013, 7, 508-510.

24 R. W. H. D. W. Mayo, F. A. Miller, Course Notes on the Interpretation of Infrared and Raman Spectra, John Wiley \& Sons, Inc., Hoboken, NJ, USA, 2003.

25 V. Amendola, O. M. Bakr and F. Stellacci, Plasmonics, 2010, 5, 85.

26 M. Abb, Y. Wang, N. Papasimakis, C. H. De Groot and O. L. Muskens, Nano Lett., 2014, 14, 346-352.

27 S. E. J. Bell and M. R. McCourt, Phys. Chem. Chem. Phys., 2009, 11, 7455.

28 D. C. Ratchford, A. D. Dunkelberger, I. Vurgaftman, J. C. Owrutsky and P. E. Pehrsson, Nano Lett., 2017, 17, 6047-6055.

29 S. Tan, A. Argondizzo, J. Ren, L. Liu, J. Zhao and H. Petek, Nat. Photonics, 2017, 11, 806-812. 